\title{
Wedelolactone disrupts the interaction of EZH2-EED complex and inhibits PRC2-dependent cancer
}

\author{
Huiming Chen ${ }^{1,2}$, Shijuan Gao ${ }^{1}$, Jiandong Li $^{1}$, Dong Liu' ${ }^{1}$, Chunjie Sheng ${ }^{1}$, Chen \\ Yao $^{1,2}$, Wei Jiang ${ }^{1}$, Jiaoxiang Wu ${ }^{1,2}$, Shuai Chen ${ }^{1,3}$ and Wenlin Huang ${ }^{1,3,4}$ \\ ${ }^{1}$ CAS Key Laboratory of Pathogenic Microbiology and Immunology, Institute of Microbiology, Chinese Academy of Sciences, \\ Beijing 100101, China \\ 2 School of Life Sciences, Anhui University, Hefei 230039, China \\ ${ }^{3}$ Sun Yat-sen University Cancer Center, State Key Laboratory of Oncology in South China, Collaborative Innovation Center \\ for Cancer Medicine, Guangzhou 510060, China \\ ${ }^{4}$ The Key Laboratory of Tumor Targeted Medicine in Guangdong Province, Guangzhou Double Bio-product Inc., Guangzhou \\ 510663, China \\ Correspondence to: Wenlin Huang, email:wl_huang@hotmail.com
}

Shuai Chen, email: shuaichen2010@hotmail.com

Keywords: epigenetic cancer therapy, surface plasmon resonance, cell cycle, apoptosis, cell migration

Received: December 18, $2014 \quad$ Accepted: March 18, $2015 \quad$ Published: April 12, 2015

This is an open-access article distributed under the terms of the Creative Commons Attribution License, which permits unrestricted use, distribution, and reproduction in any medium, provided the original author and source are credited.

\section{ABSTRACT}

Polycomb repressive complex 2 (PRC2), which is responsible for the trimethylation of H3K27 (H3K27me3), plays a part in tumorigenesis, development and/or maintenance of adult tissue specificity. The pivotal role of PRC2 in cancer makes it a therapeutic target for epigenetic cancer therapy. However, natural compounds targeting the enhancer of zeste homolog 2 (EZH2) - embryonic ectoderm development (EED) interaction to disable PRC2 complex are scarcely reported. Here, we reported the screening and identification of natural compounds which could disrupt the EZH2-EED interaction. One of these compounds, wedelolactone, binds to EED with a high affinity $(K D=2.82 \mu \mathrm{M})$, blocks the EZH2-EED interaction in vitro, induces the degradation of PRC2 core components and modulates the expression of detected PRC2 downstream targets and cancer-related genes. Furthermore, some PRC2-dependent cancer cells undergone growth arrest upon treatment with wedelolactone. Thus, wedelolactone and its derivatives which target the EZH2-EED interaction could be candidates for the treatment of PRC2-dependent cancer.

\section{INTRODUCTION}

Cancer is a major public health problem in the world. In the United States, estimated new cancer cases and cancer deaths in 2014 are 1,665,540 and 585,720, respectively [1]. Recurrent somatic mutations in numerous epigenetic regulators in various cancers draw much attention and highlight the fact that we have now entered an era of epigenetic cancer therapies [2, 3]. The epigenomic landscape features different machinery in transcriptionally active versus silent regions [4]. Polycomb group $(\mathrm{PcG})$ proteins, conserved chromatin proteins, are widely deployed in higher eukaryotes to implement gene silencing [5].

Polycomb group (PcG) proteins found in
Drosophila melanogaster are responsible for homeotic gene (Hox) silencing [6]. Further study discovered a variety of their functions such as participating in mammalian X-chromosome inactivation and imprinting $[7,8]$, maintenance of pluripotency and self-renewal in embryonic stem cells (ESCs) [9], epigenetic cell cycle control [10], cell fate decisions and developmental controls. PcG proteins mainly function by forming two evolutionarily conserved multimeric protein complexes, Polycomb repressive complexes 1 (PRC1) and Polycomb repressive complexes 2 (PRC2). They are involved in monoubiquitylation of lysine 119 of histone H2A (H2AK119ub) and di- and tri-methylation of lysine 27 of histone $\mathrm{H} 3$ (H3K27me3), respectively [11]. PRC2 contains three essential subunits: a catalytic subunit with 
methyltransferase activity, enhancer of zeste homolog 2 (EZH2) and two noncatalytic subunits, suppressor of zeste 12 (SUZ12) and embryonic ectoderm development (EED). Much attention is paid to their association with sorts of cancers like colon cancer, breast cancer, leukemia, hepatocellular carcinoma and tongue cancer [12-15].

Some groups target PRC2 through inhibiting its core component EZH2. Lots of EZH2 inhibitors are developed including 3-deazaneplanocin A (DZNep), EPZ005687 and GSK126 [16-18]. Others target PRC2 by disrupting the interaction between EED and EZH2. Interaction between EED and EZH2, which is essential to PRC2's HMTase activity as well as its function [19], serves as an interesting target for drug development. The N-terminal sequence of EZH2 (residues 39-68) mediates its association with EED, among which F42, N45, L56 and V68 are indispensable [20]. An stabilized a-helix of EZH2 (SAH-EZH2) peptide derived from this region (contains residues 40-68) was reported to selectively inhibit $\mathrm{H} 3$ Lys27 trimethylation by disrupting the EZH2-EED complex [21]. However, natural compounds targeting the EZH2-EED interaction are scarcely reported.

In this study, we used the Biacore 3000 and competitive co-immunoprecipitation (co-IP) assay to screen for small-molecule inhibitors which could disturb the binding of EZH2 to EED from the natural products library. Two compounds, epigallocatechingallate (EGCG) and wedelolactone, were identified and further studied. Interestingly, EGCG has been reported by Subhasree Roy Choudhury's group with a function to negatively regulate PRC2 [22]. In addition to disrupt PRC2, we found that wedelolactone also induce the degradation of PRC2 core components and modulate the expression of PRC2 targets and cancer-related genes. Moreover, we observed that wedelolactone could inhibit the proliferation and migration, induce cell cycle arrest and apoptosis of PRC2 dependent cancer cells. Our results provide evidences that EZH2-EED interaction is a target for the treatment of PRC2-dependent cancer and wedelolactone is a candidate for modifications in the future.

\section{RESULTS}

\section{Screen for natural compounds disrupting the EED-EZH2 interaction}

EED was reported to bind the $\mathrm{N}$-terminal sequence of EZH2 (residues 39-68) [20], so natural compounds which could bind to EED might disrupts the EZH2-EED interaction. Then we used the SPR platform Biacore 3000 to screen for natural compounds that bind to EED. Fresh recombinant EED was covalently immobilized on a CM5 sensor chip as ligand before detection. Natural compounds were diluted in PBS buffer and injected as analyte. The response unit (RU) of each compound was collected and was showed in Figure 1A.

Then, we performed competitive coimmunoprecipitation (co-IP) experiments to identify EED-EZH2 disruptors among natural compounds with RU higher than 50. In these disruptors, we found that $1 \mathrm{E} 7$ (EGCG) and 2D7 (wedelolactone) with the concentration of $5 \mu \mathrm{M}$ could disrupt the interaction between EZH2 and EED significantly (Figure 1B). In order to exclude the potential influence of other proteins in the process, we translated Myc-EZH2 and Myc-His-EED in vitro using the reticulocyte lysate system and performed competitive co-IP assays to investigate the effects of 2D7 on the interaction between EZH2 and EED. The results showed that 2D7 blocked the binding of EZH2 to EED efficiently (Figure 1C), suggesting a direct inhibition of 2D7 on the association of these two proteins.

As dismantling the PRC2 complex could result in the decrease of protein stability and further depletion of PcG members [21], we examined whether wedelolactone treatment altered the levels of EZH2 and EED. As shown in Figure 1D, wedelolactone treatment reduced the protein levels of these two core PRC2 components in human hepatocellular carcinoma cell lines HepG2, human monocytic leukemia cells THP1 and human myeloid leukemia cell lines K562.

\section{SPR detection of EGCG and wedelolactone binding to EED}

Drug candidate is usually expected to bind its target with a high affinity [23]. Here SPR platform Biacore 3000 was used to monitor the direct interaction between wedelolactone/EGCG and EED. Fresh recombinant EED proteins were covalently immobilized on a dextran sensor chip as ligand before detection. Wedelolactone/ EGCG was serially diluted in a vehicle of $1 \%$ DMSO in PBS buffer and injected as analyte to flow liquid phase. The sensorgrams had shown direct binding between wedelolactone (Figure 2A)/EGCG (Figure 2B) and EED molecule in a dose-dependent manner. Evaluated by BIA evaluation software, the equilibrium dissociation constant $\left(K_{D}\right)$ value of wedelolactone (2D7)/EGCG (1E7) to EED is $2.82 \mu \mathrm{M}$ and $15.1 \mu \mathrm{M}$, respectively (Table 2 ).

\section{Wedelolactone modulates PRC2 targets and tumor-related genes expression in PRC2- dependent cancer cells}

PRC2 has been reported to participate in silencing a myriad of target genes which are important in tumorigenesis and cancer progression. Several PRC2 target genes harbor tumor suppression function. For example, the DOC-2/DAB2 interactive protein $(D A B 2 I P)$, a growth inhibitor, is involved in the tumor necrosis factor- 
mediated JNK signaling pathway leading to cell apoptosis [24]; Beta-2 adrenergic receptor(ADRB2) activation in xenograft mouse models inhibits prostate cancer tumor growth in vivo [25]; Loss of $C D K N 2 A$ contributes to the loss of control over cell cycle, the bypass of critical senescent signals and is associated with progression to malignant disease [26] and GADD45A (growth arrest and DNA-damage-inducible, alpha) involves in cell cycle and apoptosis [27].

Dysregulation of EZH2 alters the expression of many cancer related genes [28, 29]. For instance, targeting EZH2 could deplete HOXA9 and Meis 1 levels in THP1 cells and disrupt the biological synergy between the two genes in inducing myeloid leukemia [30]. Moreover, sensitivity of cancer cells to the EZH2 inhibitors is partly dependent on PTEN and p53 [31, 32].

To explore the regulation of wedelolactone treatment on the expression of these PRC2 target genes and cancer related genes, HepG2, THP1 and K562 cells were treated with $50 \mu \mathrm{M}$ wedelolactone for $24 \mathrm{~h}$. Total RNA were extracted and the mRNA levels of the above genes were analyzed by quantitative real-time PCR. As shown in Figure $3 \mathrm{~A}$, wedelolactone treatment significantly induces the expression of GADD45A, DAB2IP, ADRB2, CDKN2A and p53 while represses Meis 1 expression in HepG2 cells. As shown in Figure 3B, wedelolactone significantly repressed HOXA9 and Meis 1 expression while enhances the expression of GADD45A and p53 in THP1 cells. At the meantime, the expression of GADD45A, PTEN and p53 were activated after treatment with wedelolactone in

A

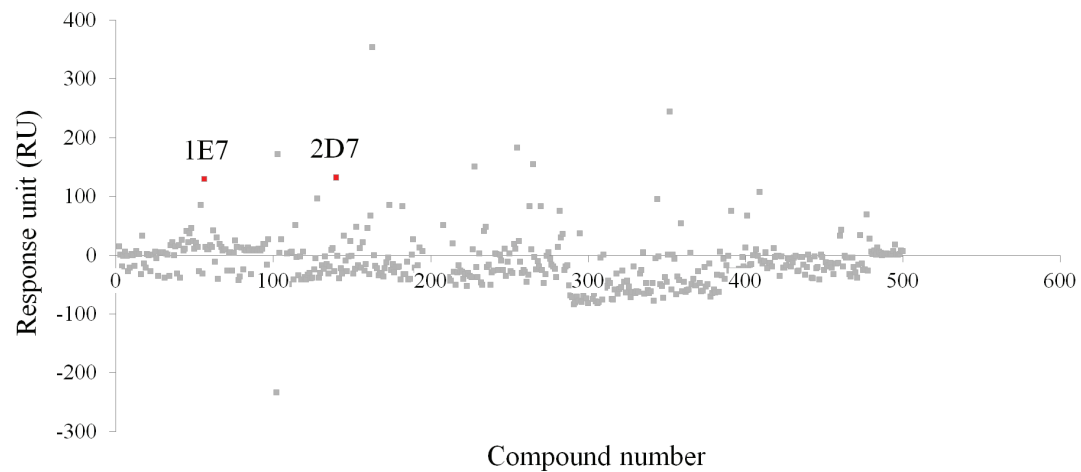

B

$\mathrm{C}$

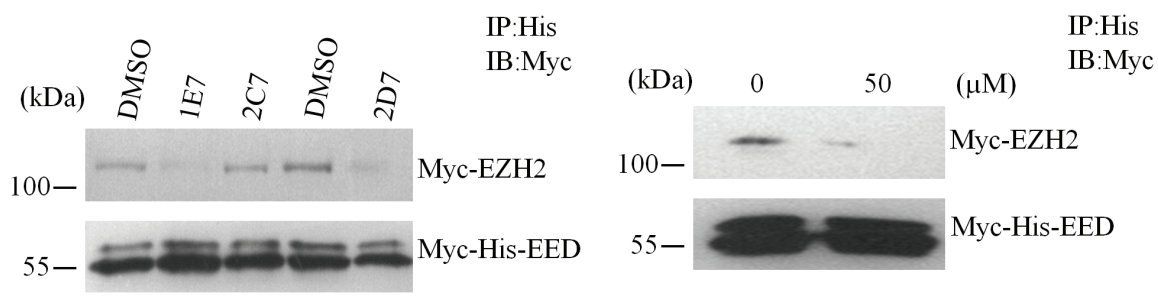

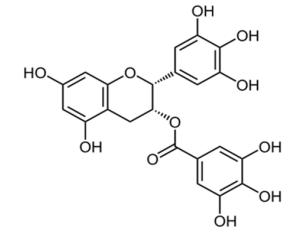

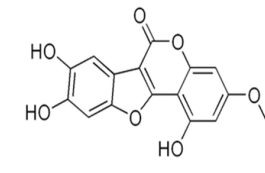

1E7 (Epigallocatechin gallate) 2D7 (Wedelolactone)

$\mathrm{D}$

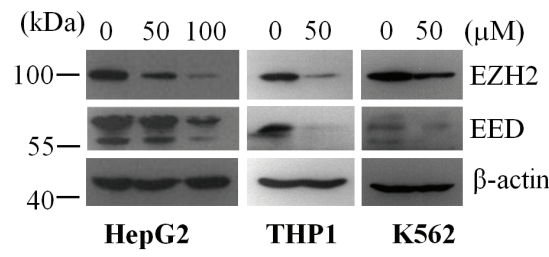

Figure 1: Screen for natural compounds disrupting the EED-EZH2 interaction. (A) Representative sensorgrams were obtained from injections of natural compounds over the CM5-EED surface. 1E7 and 2D7 refers to epigallocatechingallate and wedelolactone, respectively. (B) Competitive co-immunoprecipitation assay was performed with the indicated natural compounds with the concentration of $5 \mu \mathrm{M}$ or DMSO. 2C7 refers to tetrandrine as a negative control. The protein levels of Myc-EZH2 and Myc-His-EED were evaluated by WB with anti-Myc antibody. (C) Myc-EZH2 and Myc-His-EED were translated with the reticulocyte lysate system in vitro followed by incubation with wedelolactone or DMSO to perform competitive co-IP analysis. (D) Wedelolactone depletes PcG proteins. HepG2, THP1 and K562 cells were incubated with the indicated concentrations of wedelolacone for $24 \mathrm{~h}$. The levels of EZH2, EED and H3K27me3 were then analyzed with specific antibodies as indicated. 
A

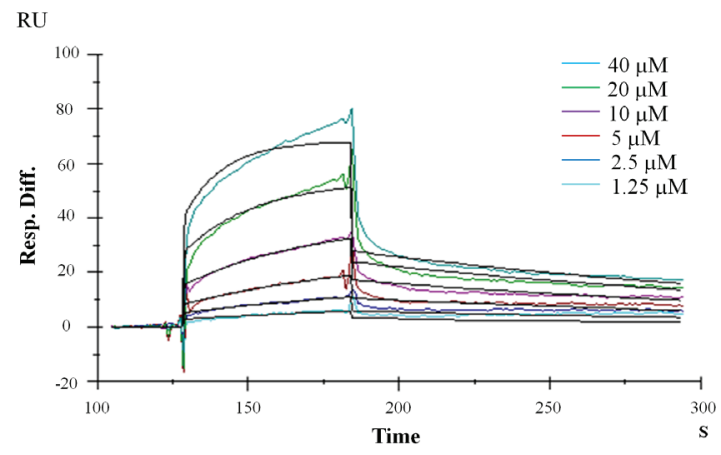

B

2D7

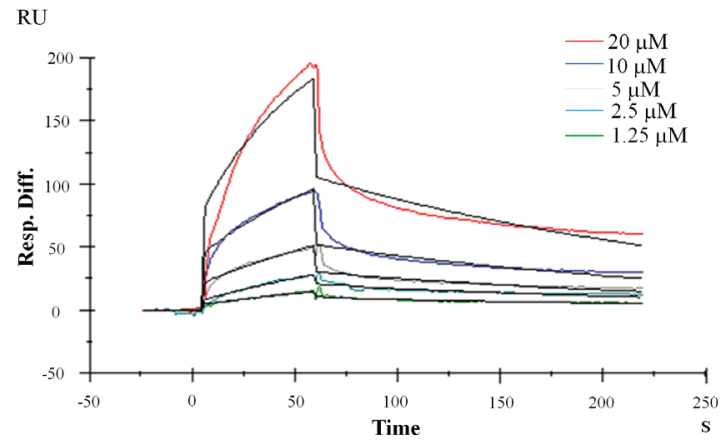

$1 \mathrm{E} 7$

Figure 2: Kinetics analysis of wedelolactone and EGCG binding to EED based on SPR platform Biacore 3000. Representative Sensorgrams were obtained from injections of wedelolactone (A) or EGCG (B) at indicated concentrations.

A

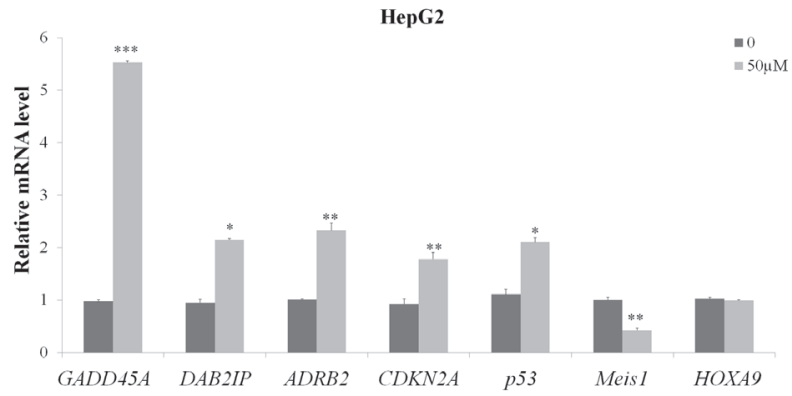

B

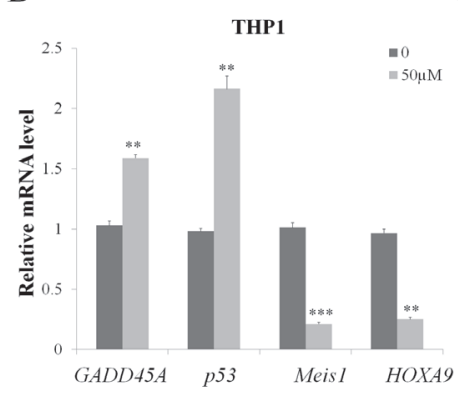

$\mathrm{C}$

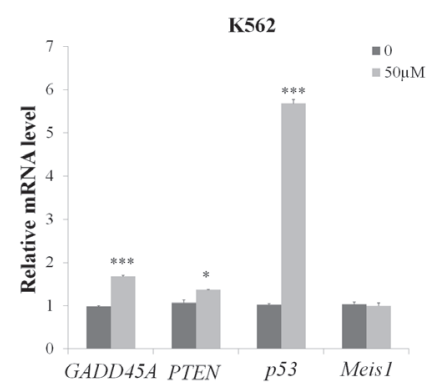

Figure 3: Wedelolactone modulates PRC2 target and tumor-related genes expression in PRC2-dependent cancer cells. HepG2 cells (A), THP1 cells (B) and K562 cells (C) were treated with $50 \mu \mathrm{M}$ wedelolactone for $24 \mathrm{~h}$. Total RNA was isolated and qRT-PCR was performed with specific primers for the indicated target genes. Quantification results were shown as folds of control and expressed as the mean $\pm \mathrm{SD}(n=3) . * P<0.05, * * P<0.01, * * * P<0.001$. 
Table 1: Oligonucleotide sequences used in this study

\begin{tabular}{|c|c|}
\hline Primer name & Primer sequence \\
\hline GAPDH F1 & GAAGGTGAAGGTCGGAGTC \\
\hline GAPDH R1 & GAAGATGGTGATGGGATTC \\
\hline DAB2IP F1 & ACATCCAGATGAAGGGCATC \\
\hline DAB2IP R1 & GCGTGGTCCTTCTTCTTCAGTTC \\
\hline ADRB2 F1 & AGCCAGTGCGCTCACCTGCCAGACT \\
\hline ADRB2 R1 & GCTCGAACTTGGCAATGGCTGTGA \\
\hline CDKN2A F1 & GCTGCCCAACGCACCGAATA \\
\hline CDKN2A R1 & ACCACCAGCGTGTCCAGGAA \\
\hline PTEN F1 & AATCCTCAGTTTGTGGTCT \\
\hline PTEN R1 & GGTAACGGCTGAGGGAACT \\
\hline Meis1 F1 & CCCTGGAATGCCAATGTCA \\
\hline Meis1 R1 & GAGCGTGAATGTCCATGACTTG \\
\hline P53 F1 & CCAGCAGCTCCTACACCGGC \\
\hline P53 R1 & GAAACCGTAGCTGCCCTG \\
\hline GADD45A F1 & CGCCTGTGAGTGAGTGC \\
\hline GADD45A R1 & CTTATCCATCCTTTCGGTCTT \\
\hline HOXA9 F1 & GCTTGTGGTTCTCCTCCAGT \\
\hline HOXA9 R1 & CCAGGGTCTGGTGTTTTGTA \\
\hline
\end{tabular}


Table 2: Kinetic parameters of the binding of 2D7 and 1E7 to EED.

\begin{tabular}{cccccc}
\hline & $R_{\max }(\mathrm{RU})$ & $k_{\text {on }}\left(\mathrm{M}^{-1} \mathrm{~s}^{-1}\right)$ & $k_{\text {off }}\left(\mathrm{s}^{-1}\right)$ & $K_{D}(\mathrm{M})$ & $\chi^{2}$ \\
\hline $2 \mathrm{D} 7$ & 30.2 & $1.82 \times 10^{3}$ & $5.13 \times 10^{-3}$ & $2.82 \times 10^{-6}$ & 2.55 \\
\hline $1 \mathrm{E} 7$ & 414 & 302 & $4.57 \times 10^{-3}$ & $1.51 \times 10^{-5}$ & 20.7 \\
\hline
\end{tabular}

$R_{\text {max }}$, maximum analyte binding capacity; $k_{\text {on }}$, association rate constant; $k_{\text {off }}$,

dissociation rate constant; $K_{D}$, equilibrium dissociation constant. $K_{D}=k_{\text {off }} / k_{o n}$;

$\chi 2$,statistical value in Biacore. All parameters were measured according to Langmuir

fitting model, using Biacore Evaluation Software (version 4.01).

leukemia cell lines K562 (Figure 3C). Together, our results indicated that PRC2 targets and tumor-related genes which were involved in apoptosis and cell cycle arrest were modulated by wedelolactone.

\section{Wedelolactone inhibits PRC2-dependent cancer cells}

Since human leukemia K562 cells, THP1 monocytes and hepatocellular carcinoma HepG2 cells are partially PRC2-dependent [21, 33, 34], we studied the anti-cancer effect of wedelolactone on these cells.

We first examined the effects of wedelolactone on cell proliferation. As shown in Figure 4A, $50 \mu \mathrm{M}$ of wedelolactone treatment repressed the proliferation of HepG2, THP1 and K562 cells. Since many drugs have been shown to inhibit cancer cells through induction of apoptosis, we then detected the apoptotic ratio in cells with or without wedelolactone treatment by Annexin V-FITC/PI double staining assay. As shown in Figure $4 \mathrm{~B}$, the presence of wedelolactone significantly increased apoptosis in HepG2, THP1 and K562 cells.

It is well-known that most anticancer agents, such as vinblastine and paclitaxel, have been documented to arrest cell cycle [35]. So we examined the effect of wedelolatctone on cell cycle distribution of HepG2, THP1 and K562 cells. Compared with control, there was an accumulation of cell population in $\mathrm{S}$ and G2/M phase after wedelolactone exposure in HepG2 cells and the proportion of $\mathrm{K} 562$ cells in S phase were increased after wedelolactone exposure (Figure 4C). However, it exhibited no significant effect on THP1 cells.

Recently, several studies about EZH2 regulating cell invasion in various types of cancer showed that one of the major EZH2 PRC2-dependent function is promoting cell invasion [36-38]. Additionally, the migration of K562 and THP1 cells are scarcely reported. So we interrogated the effect of wedelolactone on the migration of HepG2 cells.

To determine whether wedelolactone inhibits cell migration in HepG2 cells, we performed Transwell migration assays. As shown in Figure 4D, the presence of wedelolactone significantly suppressed cell migration in HepG2 cells. Together, our data suggested that wedelolactone inhibited proliferation, induced apoptosis and cell cycle arrest and suppressed cell migration of PRC2-depedent cancer cells.

\section{DISCUSSION}

Wedelolactone is an essential active compound of Eclipta prostrate. It has been reported to possess various biological functions, including the inhibition of IKK kinase, $\mathrm{K}+$-ATPase activity, hepatitis virus $\mathrm{C}$ RNA-polymerase, phospholipase A2 , 5-lipoxygenase and DNA topoisomerase II $\alpha$ [39]. Also, it exhibits anticancer function in some cancers, such as prostate cancer, breast cancer and so on [39]. The growth inhibition effects of wedelolactone on tumor cells were believed to be accomplished through its inhibition of IKK, the androgen receptor [40], or topoisomerase II. But its function on the EED-EZH2 interaction and the PRC2 activity is unknown.

In the present study, we present a new method to identify inhibitors targeting PRC2. Firstly, Biacore 3000 was used to screen for natural compouds which could bind to EED. Then the competitive co-IP experiment was performed to further identify PRC2 disruptors. By this way, we identified that EGCG and wedelolactone could bind to EED and target the EZH2-EED interaction.

Tumor suppressors refer to a large group of molecules that can prevent cancer through controlling cell 


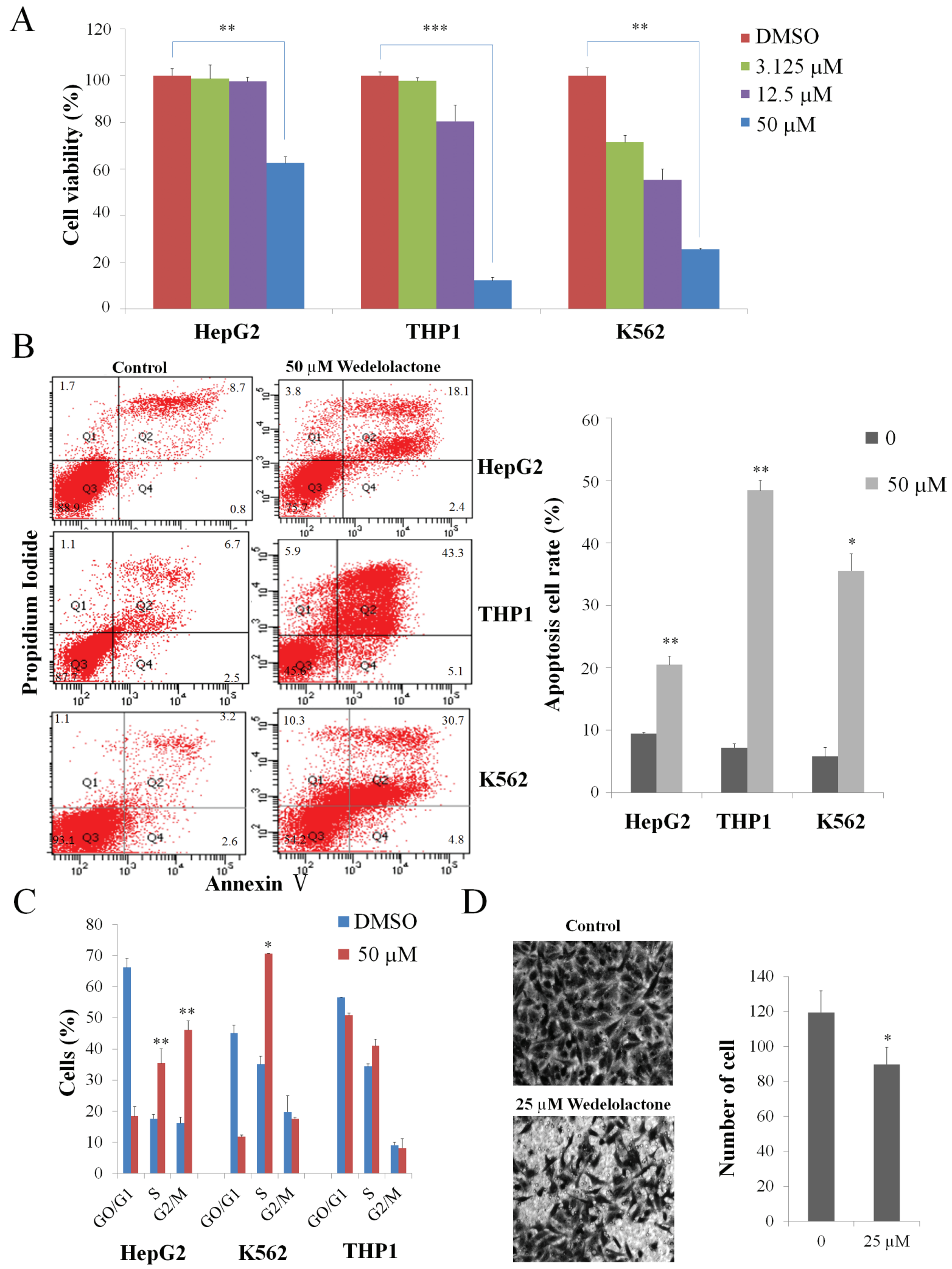

Figure 4: Wedelolactone inhibits PRC2-dependent cancer cells. K562 cells, HepG2 cells and THP1 cells were treated for 48 h with the indicated concentrations of wedelolactone. The cells were then harvested. The viability was assayed by MTT assay (A) and the apoptosis was assayed by AnnexinV-FITC/PI double staining assay (B). (C) Cells were treated with $50 \mu \mathrm{M}$ wedelolactone for $24 \mathrm{~h}$, and the cell cycle distribution was subsequently determined via flow cytometric analysis. (D) wedelolactone regulates migration of HepG2 cells. Wedelolactone decreased the number of migration cells compared with control cells (original magnification $\times 200$ ). Quantification results were shown as folds of control and expressed as the mean $\pm \mathrm{SD}(n=3) . * P<0.05, * * P<0.01, * * * P<0.001$. 
division, promoting apoptosis, helping DNA damage repair and suppressing metastasis [41]. So reactivating tumor suppressors which are silenced by PRC2 will contribute to the inhibition of carcinoma cells proliferation. Indeed, wedelolactone could activate PRC2 downstream tumor suppression genes such as DAB2IP, ADRB2,CDKN2A and $G A D D 45 A$ (Figure $3 \mathrm{~A}$ ), thus it serves as a mechanism for its inhibition on PRC2-dependent cancer cells. In fact, not all the target genes can be influenced by wedelolactone (data not shown).

In conclusion, we identified that wedelolactone could bind to EED and target PRC2, thereby modulate its targets and cancer-related genes. As a consequence, wedelolactone exhibits anti-cancer effects by inducing proliferation and migration inhibition, apoptosis and cell cycle arrest of PRC2-dependent cancer cells. So it could serve as a candidate for the treatment of PRC2dependent cancer. Also, our work verified the possibility to the development of anti-cancer agents by disrupting the association of core PRC2 components EZH2 and EED.

\section{MATERIALS AND METHODS}

\section{Plasmids}

The EZH2 gene was amplified by PCR and subcloned into pcDNA3.0-Myc (Invitrogen). The EED gene was amplified by PCR and subcloned into pcDNA4.0-Myc/His (Invitrogen) and pGEX-4T-1 plasmid (GE Healthcare) respectively.

\section{Abs and reagents}

The primary antibodies used in this study were as follows: anti-Myc (sc-40), anti-EED (sc-28701) and anti- $\beta$-actin (sc-47778) were purchased from Santa Cruz Biotechnology. Anti-EZH2 (\#3147S) was from Cell Signaling Technology, anti-His (\#TA-02) was from ZSGB-BIO and anti-trimethyl histone H3 (Lys27) antibody (ABE44-S) was from Millipore. Wedelolactone was purchased from National Institutes for Food and Drug Control (NIFDC, China). Dimethylsulfoxide (DMSO) was obtained from Sigma-aldrich (USA). Protein G beads and GST beads were purchased from Santa Cruz Biotechnology and GE Healthcare, respectively.

\section{Cell culture}

HepG2, K562 and 293T cells were cultured in DMEM (Invitrogen) supplemented with 10\% FBS (Hyclone), $100 \mathrm{U} / \mathrm{ml}$ penicillin and $100 \mu \mathrm{g} / \mathrm{ml}$ streptomycin. THP1 cells were cultured in RPMI-1640 supplemented with $10 \% \mathrm{FBS}, 100 \mathrm{U} / \mathrm{ml}$ penicillin, 100 $\mu \mathrm{g} / \mathrm{ml}$ streptomycin and $2 \mathrm{mM}$ L-Glutamine. All cell lines were maintained at $37^{\circ} \mathrm{C}$ under a $5 \% \mathrm{CO}_{2}$ atmosphere. Transfection of cells was performed by using Entranster-H (Engreen, China) according to the manufacturer's instructions.

\section{Binding detection based on SPR platform}

The interaction between compound and protein was detected by surface plasmon resonance platform Biacore 3000 (GE Healthcare). Fresh EED protein was diluted to $100 \mu \mathrm{g} / \mathrm{ml}$ in $10 \mathrm{mM}$ acetate buffer ( $\mathrm{pH} 5.0)$, and then immobilized as ligand in the NHS/EDC pre-activated CM5 sensor chip, following blocking by ethanolamine. Final amount of protein immobilization reached 10000 RU. The compound stock was diluted in a vehicle of $1 \%$ DMSO (v/v) in phosphate buffered saline (PBS). The dilutions were injected as analyte flow liquid phase with PBS containing $1 \%$ DMSO $(\mathrm{v} / \mathrm{v})$ as running buffer at a constant flow rate of $30 \mu \mathrm{l} / \mathrm{min}$. Ninety seconds' association time was set, followed by $180 \mathrm{~s}$ dissociation time. All buffers in the experiment were subjected to be filtered by $0.22 \mu \mathrm{m}$ filters and degassed by ultrasonic. The data were collected by Biacore Control Software (version 4.1.1). Kinetics and affinity parameters were evaluated in Langmuir model (1:1) by using BIA evaluation software (version 4.1).

\section{Competitive co-immunoprecipitation assay}

Cell lysates from 293T cells transfected with MycEZH2 and Myc-His-EED were incubated with anti-His antibody, protein $\mathrm{G}$ beads and natural compound with corresponding concentration or DMSO overnight at $4{ }^{\circ} \mathrm{C}$. The beads were then washed three times and boiled to be used for WB.

\section{In vitro translation assays}

Myc-EZH2 and Myc-His-EED were translated in vitro with TNT T7 coupled reticulocyte lysate system (Promega, \#L4611) according to the manufacturer's instructions. The in vitro translated products were used to perform competitive co-immunoprecipitation assay.

\section{Western blotting}

Cells were lysed and prepared with $1 \mathrm{X}$ SDS Reducing sample buffer (CST, \#7722) according to the manufacturer's instructions. Appropriate volume of sample was loaded onto the SDS-polyacrylamide gels and transferred to a PVDF membrane. After blocking, the membrane was incubated with the primary antibody overnight at $4^{\circ} \mathrm{C}$ followed by incubation with a horseradish 
peroxidase-conjugated secondary antibody for $2 \mathrm{~h}$ at room temperature. Bands were detected using enhanced chemiluminescence (Applygen, China).

\section{RNA extraction and quantitative reverse transcriptase polymerase chain reaction (qRT- PCR)}

Total RNA was isolated from the cells using TRNzol (TIANGEN, China). cDNA was synthesized using the RevertAid First Strand cDNA Synthesis kit (Thermo Scientific, \#K1622). Quantitative real-time PCR (qRTPCR) was conducted using SYBR premix Ex Taq II (Takara, China). Thermal cycling was performed using an ABI 7300 real-time PCR machine (Applied Biosystems) as follows: $95^{\circ} \mathrm{C}$ for $30 \mathrm{~s}$ followed by 40 cycles of amplification for $5 \mathrm{~s}$ at $95^{\circ} \mathrm{C}, 31 \mathrm{~s}$ at $60^{\circ} \mathrm{C}$. The primer sequences used for PCR are listed in Table 1.

\section{Measurement of cell viability}

Cell viability was determined by MTT Cell Proliferation and Cytotoxicity Detection Kit (KGA312, KeyGEN BioTECH, China). A total of $5 \times 10^{3}$ cells were seeded in 96 well plates. At $48 \mathrm{~h}$ post-treatment with different concentrations of wedelolactone, $1 \times$ MTT was added to the wells and incubated for additional $4 \mathrm{~h}$ at $37^{\circ} \mathrm{C}$. The optical density of the dissolved material was measured at $490 \mathrm{~nm}$.

\section{Cell cycle analysis}

The effect of wedelolactone on cell cycle distribution was determined by flow cytometric analysis. The cells were treated with $50 \mu \mathrm{M}$ wedelolactone for 24 h. Appropriate controls were also set up. After treatment, $1 \times 10^{5}$ floating and adherent cells were collected, washed with PBS and fixed with $70 \%$ ethanol. Staining for DNA content was performed using Cell Cycle Detection Kit (KGA512, KeyGEN BioTECH, China). Populations in $\mathrm{G} 0 / \mathrm{G} 1, \mathrm{~S}$ and $\mathrm{G} 2 / \mathrm{M}$ phases were measured by BD FACSCalibur Flow Cytometry System with CellQuest Pro software (BD Bioscience). Data were analyzed using the ModFit 3.0 Software.

\section{Detection of apoptotic cells by flow cytometry}

Cells were plated in six-well plates at a density of $1 \times 10^{5}$ cells $/ \mathrm{ml}$ and incubated overnight. Wedelolactone or DMSO was then added into each well and incubated for $48 \mathrm{~h}$. Cells were collected and washed with PBS, followed by resuspension in $300 \mu \mathrm{l}$ binding buffer at a concentration of $5 \times 10^{5}$ cells $/ \mathrm{ml}$. Mixed with Annexin V-FITC and propidium iodide (PI) according to the manufacturer's instructions. The mixed solution was incubated in the dark at room temperature for $15 \mathrm{~min}$. Cell apoptosis analysis was performed using the BD FACSAriaII Flow Cytometry System (BD, USA) within $1 \mathrm{~h}$. Data were analyzed using the FACSDiva Version 6.1 Software.

\section{Cell migration assay}

HepG2 cells were treated with $25 \mu \mathrm{m}$ wedelolactone or DMSO for $12 \mathrm{~h}$ then the cells were trypsinized and replated onto the upper chamber of a Transwell filter with $8 \mu \mathrm{m}$ pores (Costar) at $2 \times 10^{5}$ cells/well in serumfree medium. Medium supplemented with 10\% FBS was placed in the bottom well, and the cells were then incubated for $24 \mathrm{~h}$ at $37^{\circ} \mathrm{C}$ in a humidified $5 \% \mathrm{CO}_{2}$ atmosphere. After the incubation, the chambers were removed, and migration cells on the bottom side of the membrane were fixed with methanol for $15 \mathrm{~min}$ and stained with gentian violet for $10 \mathrm{~min}$. Each experiment was performed in triplicate, and the number of cells in five random fields on the underside of the filter was counted and averaged. The results were expressed as the migrated cell number.

\section{Statistical analysis}

The data are presented as mean \pm stand deviation (S.D.). Parametrical data were compared using Student's $t$ test. One-way ANOVA analysis was used to determine the difference between independent groups. The differences between the variants were considered to be statistically significant if $P<0.05$.

\section{ACKNOWLEDGEMENTS}

This work was supported by National Basic Research Program of China (973 Program) (No. 2012CB518900,2011CB504706 and 2011CB504805), the National Natural Science Foundation of China (No. 81171572) and Guangdong Innovative Research Team Program (No.2009010058). We are grateful to Mrs. Lanqing Ma (Institute of Microbiology, Chinese Academy of Sciences) for her technical assistance. We thank Qianfei Jeffery Wang from Beijing Institute of Genomics Chinese Academy of Sciences for his kindly offer of THP1 cells.

\section{CONFLICTS OF INTEREST STATEMENT}

The authors declare no conflict of interest.

\section{REFERENCES}

1. Siegel R, Ma J, Zou Z and Jemal A. Cancer statistics, 2014. 
CA: a cancer journal for clinicians. 2014; 64:9-29.

2. Dawson MA and Kouzarides T. Cancer epigenetics: from mechanism to therapy. Cell. 2012; 150:12-27.

3. Aumann S and Abdel-Wahab O. Somatic alterations and dysregulation of epigenetic modifiers in cancers. Biochemical and biophysical research communications. 2014.

4. Filion GJ, van Bemmel JG, Braunschweig U, Talhout W, Kind J, Ward LD, Brugman W, de Castro IJ, Kerkhoven RM, Bussemaker HJ and van Steensel B. Systematic protein location mapping reveals five principal chromatin types in Drosophila cells. Cell. 2010; 143:212-224.

5. Schwartz YB and Pirrotta V. Polycomb silencing mechanisms and the management of genomic programmes. Nature reviews Genetics. 2007; 8:9-22.

6. Schwartz YB, Kahn TG, Nix DA, Li XY, Bourgon R, Biggin $\mathrm{M}$ and Pirrotta V. Genome-wide analysis of Polycomb targets in Drosophila melanogaster. Nature genetics. 2006; 38:700-705

7. Plath K, Fang J, Mlynarczyk-Evans SK, Cao R, Worringer KA, Wang H, de la Cruz CC, Otte AP, Panning B and Zhang Y. Role of histone H3 lysine 27 methylation in X inactivation. Science. 2003; 300:131-135.

8. Umlauf D, Goto Y, Cao R, Cerqueira F, Wagschal A, Zhang $\mathrm{Y}$ and Feil R. Imprinting along the Kcnq1 domain on mouse chromosome 7 involves repressive histone methylation and recruitment of Polycomb group complexes. Nature genetics. 2004; 36:1296-1300

9. Aloia L, Di Stefano B and Di Croce L. Polycomb complexes in stem cells and embryonic development. Development. 2013; 140:2525-2534.

10. Martinez AM and Cavalli G. The role of polycomb group proteins in cell cycle regulation during development. Cell cycle. 2006; 5:1189-1197.

11. Cao R, Tsukada Y and Zhang Y. Role of Bmi-1 and Ring1A in $\mathrm{H} 2 \mathrm{~A}$ ubiquitylation and Hox gene silencing. Molecular cell. 2005; 20:845-854.

12. Gupta RA, Shah N, Wang KC, Kim J, Horlings HM, Wong DJ, Tsai MC, Hung T, Argani P, Rinn JL, Wang YL, Brzoska P, Kong B, Li R, West RB, van de Vijver MJ, et al. Long non-coding RNA HOTAIR reprograms chromatin state to promote cancer metastasis. Nature. 2010; 464:1071U1148.

13. Neff T, Sinha AU, Kluk MJ, Zhu N, Khattab MH, Stein L, Xie HF, Orkin SH and Armstrong SA. Polycomb repressive complex 2 is required for MLL-AF9 leukemia. Proceedings of the National Academy of Sciences of the United States of America. 2012; 109:5028-5033.

14. Gao SB, Xu B, Ding LH, Zheng QL, Zhang L, Zheng QF, Li SH, Feng ZJ, Wei J, Yin ZY, Hua X and Jin GH. The functional and mechanistic relatedness of EZH2 and menin in hepatocellular carcinoma. Journal of hepatology. 2014.

15. Li Z, Wang Y, Qiu J, Li Q, Yuan C, Zhang W, Wang D, Ye J, Jiang H, Yang J and Cheng J. The polycomb group protein EZH2 is a novel therapeutic target in tongue cancer. Oncotarget. 2013; 4:2532-2549.

16. Tan J, Yang X, Zhuang L, Jiang X, Chen W, Lee PL, Karuturi RK, Tan PB, Liu ET and Yu Q. Pharmacologic disruption of Polycomb-repressive complex 2-mediated gene repression selectively induces apoptosis in cancer cells. Genes \& development. 2007; 21:1050-1063.

17. Knutson SK, Wigle TJ, Warholic NM, Sneeringer CJ, Allain CJ, Klaus CR, Sacks JD, Raimondi A, Majer CR, Song J, Scott MP, Jin L, Smith JJ, Olhava EJ, Chesworth $\mathrm{R}$, Moyer MP, et al. A selective inhibitor of EZH2 blocks H3K27 methylation and kills mutant lymphoma cells. Nature chemical biology. 2012; 8:890-896.

18. McCabe MT, Ott HM, Ganji G, Korenchuk S, Thompson C, Van Aller GS, Liu Y, Graves AP, Della Pietra A, 3rd, Diaz E, LaFrance LV, Mellinger M, Duquenne C, Tian $\mathrm{X}$, Kruger RG, McHugh CF, et al. EZH2 inhibition as a therapeutic strategy for lymphoma with EZH2-activating mutations. Nature. 2012; 492:108-112.

19. Cao R, Wang L, Wang H, Xia L, Erdjument-Bromage H, Tempst P, Jones RS and Zhang Y. Role of histone H3 lysine 27 methylation in Polycomb-group silencing. Science. 2002; 298:1039-1043.

20. Han Z, Xing X, Hu M, Zhang Y, Liu P and Chai J. Structural basis of EZH2 recognition by EED. Structure. 2007; 15:1306-1315.

21. Kim W, Bird GH, Neff T, Guo G, Kerenyi MA, Walensky LD and Orkin SH. Targeted disruption of the EZH2-EED complex inhibits EZH2-dependent cancer. Nature chemical biology. 2013; 9:643-650.

22. Choudhury SR, Balasubramanian S, Chew YC, Han B, Marquez VE and Eckert RL. (-)-Epigallocatechin-3gallate and DZNep reduce polycomb protein level via a proteasome-dependent mechanism in skin cancer cells. Carcinogenesis. 2011; 32:1525-1532.

23. O'Shannessy DJ, Somers EB, Albone E, Cheng X, Park YC, Tomkowicz BE, Hamuro Y, Kohl TO, Forsyth TM, Smale R, Fu YS and Nicolaides NC. Characterization of the Human Folate Receptor Alpha Via Novel Antibody-Based Probes. Oncotarget. 2011; 2:1227-1243.

24. Chen H, Tu SW and Hsieh JT. Down-regulation of human DAB2IP gene expression mediated by polycomb Ezh2 complex and histone deacetylase in prostate cancer. The Journal of biological chemistry. 2005; 280:22437-22444.

25. Yu J, Cao Q, Mehra R, Laxman B, Yu J, Tomlins SA, Creighton CJ, Dhanasekaran SM, Shen R, Chen G, Morris DS, Marquez VE, Shah RB, Ghosh D, Varambally S and Chinnaiyan AM. Integrative genomics analysis reveals silencing of beta-adrenergic signaling by polycomb in prostate cancer. Cancer cell. 2007; 12:419-431.

26. Rayess H, Wang MB and Srivatsan ES. Cellular senescence and tumor suppressor gene p16. International journal of cancer Journal international du cancer. 2012; 130:17151725. 
27. Qi W, Chan H, Teng L, Li L, Chuai S, Zhang R, Zeng J, Li M, Fan H, Lin Y, Gu J, Ardayfio O, Zhang JH, Yan X, Fang J, Mi Y, et al. Selective inhibition of Ezh2 by a small molecule inhibitor blocks tumor cells proliferation. Proceedings of the National Academy of Sciences of the United States of America. 2012; 109:21360-21365.

28. Chinaranagari S, Sharma $\mathrm{P}$ and Chaudhary J. EZH2 dependent H3K27me3 is involved in epigenetic silencing of ID4 in prostate cancer. Oncotarget. 2014; 5:7172-7182.

29. Pasini D, Bracken AP and Helin K. Polycomb group proteins in cell cycle progression and cancer. Cell cycle. 2004; 3:396-400.

30. Fiskus W, Pranpat M, Balasis M, Herger B, Rao R, Chinnaiyan A, Atadja P and Bhalla K. Histone deacetylase inhibitors deplete enhancer of zeste 2 and associated polycomb repressive complex 2 proteins in human acute leukemia cells. Molecular cancer therapeutics. 2006; 5:3096-3104.

31. Benoit YD, Witherspoon MS, Laursen KB, Guezguez A, Beausejour M, Beaulieu JF, Lipkin SM and Gudas LJ. Pharmacological inhibition of polycomb repressive complex-2 activity induces apoptosis in human colon cancer stem cells. Experimental cell research. 2013; 319:14631470 .

32. Cheng LL, Itahana Y, Lei ZD, Chia NY, Wu Y, Yu Y, Zhang SL, Thike AA, Pandey A, Rozen S, Voorhoeve PM, Yu Q, Tan PH, Bay BH, Itahana K and Tan P. TP53 genomic status regulates sensitivity of gastric cancer cells to the histone methylation inhibitor 3-deazaneplanocin A (DZNep). Clinical cancer research : an official journal of the American Association for Cancer Research. 2012; 18:4201-4212.

33. Lin YH, Lee CC, Chang FR, Chang WH, Wu YC and Chang JG. 16-hydroxycleroda-3,13-dien-15,16-olide regulates the expression of histone-modifying enzymes PRC2 complex and induces apoptosis in CML K562 cells. Life sciences. 2011; 89:886-895.

34. Chiang CW, Huang Y, Leong KW, Chen LC, Chen HC, Chen SJ and Chou CK. PKCalpha mediated induction of miR-101 in human hepatoma HepG2 cells. Journal of biomedical science. 2010; 17:35.

35. Newman DJ, Cragg GM, Holbeck S and Sausville EA. Natural products and derivatives as leads to cell cycle pathway targets in cancer chemotherapy. Current cancer drug targets. 2002; 2:279-308.

36. Kleer CG, Cao Q, Varambally S, Shen RL, Ota L, Tomlins SA, Ghosh D, Sewalt RGAB, Otte AP, Hayes DF, Sabel MS, Livant D, Weiss SJ, Rubin MA and Chinnaiyan AM. EZH2 is a marker of aggressive breast cancer and promotes neoplastic transformation of breast epithelial cells. Proceedings of the National Academy of Sciences of the United States of America. 2003; 100:11606-11611.

37. Rao ZY, Cai MY, Yang GF, He LR, Mai SJ, Hua WF, Liao YJ, Deng HX, Chen YC, Guan XY, Zeng YX, Kung HF and Xie D. EZH2 supports ovarian carcinoma cell invasion and/ or metastasis via regulation of TGF-betal and is a predictor of outcome in ovarian carcinoma patients. Carcinogenesis. 2010; 31:1576-1583.

38. Cao Q, Yu J, Dhanasekaran SM, Kim JH, Mani RS, Tomlins SA, Mehra R, Laxman B, Cao X, Yu J, Kleer CG, Varambally $\mathrm{S}$ and Chinnaiyan AM. Repression of E-cadherin by the polycomb group protein EZH2 in cancer. Oncogene. 2008; 27:7274-7284.

39. Benes P, Knopfova L, Trcka F, Nemajerova A, Pinheiro D, Soucek K, Fojta M and Smarda J. Inhibition of topoisomerase IIalpha: novel function of wedelolactone. Cancer letters. 2011; 303:29-38.

40. Tsai CH, Lin FM, Yang YC, Lee MT, Cha TL, Wu GJ, Hsieh SC and Hsiao PW. Herbal extract of Wedelia chinensis attenuates androgen receptor activity and orthotopic growth of prostate cancer in nude mice. Clinical cancer research : an official journal of the American Association for Cancer Research. 2009; 15:5435-5444.

41. Sun W and Yang J. Functional mechanisms for human tumor suppressors. Journal of Cancer. 2010; 1:136-140. 